\title{
Mental health in the United States during the COVID-19 pandemic: A longitudinal examination of the ameliorating effect of meaning salience
}

\author{
Kristine Klussman $^{1}$ (D) - Austin Lee Nichols ${ }^{1}$ (D) $\cdot$ Julia Langer $^{1}$ (D) \\ Accepted: 23 February 2021 / Published online: 12 March 2021 \\ (C) The Author(s), under exclusive licence to Springer Science+Business Media, LLC part of Springer Nature 2021
}

\begin{abstract}
During the COVID-19 pandemic, various restrictions forced people around the world to socially isolate. People were asked to stay at home and were largely unable to do many of the activities that they derived meaning from. Since meaning is often related to mental health, these restrictions were likely to decrease mental health. The current study aimed to examine these effects and additionally benefit individuals' mental health by making their meaning salient. Specifically, the goal of the research was to design an intervention that could counter the potential negative effects of social distancing. We recruited a total of 96 U.S.A. residents $\left(M_{\text {age }}=34.45,92.7 \%\right.$ Female $)$ and assigned them to either the control group or to a meaning salience intervention. That is, participants either focused on the meaning of their daily activities $(n=45)$ or did not participate in any study-related activities during the week $(n=51)$. They completed various measures of mental health before and after this experimental period. Results suggested that the control group reported significantly greater anxiety, depression, and stress at the end of the week. In contrast, the experimental group reported less anxiety and trended toward less depression and stress at the end of that same week. In all, results suggest that simply focusing on one's daily activities and the meaning found in them protected people from the otherwise detrimental effects of the restrictions. This provides a promising and simple intervention that may assist both individuals and practitioners aiming to improve mental health, especially in challenging times.
\end{abstract}

Keywords Meaning salience $\cdot$ Meaning in life $\cdot$ Anxiety $\cdot$ Depression $\cdot$ Stress

\section{Introduction}

Researchers often consider meaning in life to be an inherent part of happiness and well-being (Heintzelman, 2018; Heintzelman et al., 2020). However, what happens when external circumstances take away one's ability to find meaning and improve well-being? Research suggests that those currently suffering experience significantly lower meaning in life (Edwards \& Van Tongeren, 2020). At the same time, suffering increases the desire for meaning in life (Edwards \& Van Tongeren, 2020). In a time of global pandemic, when people are asked to keep socially distant, what happens to one's well-

The data that support the findings of this study are available from the first author upon reasonable request.

Austin Lee Nichols

research@connectionlab.com

1 Connection Lab, San Francisco, CA, USA being, and how can we prevent these potentially deleterious effects?

During times of social isolation, it is especially important to develop methods to promote well-being. Recently, researchers have focused on making meaning in life salient and suggest promising avenues for doing so using short-term interventions (Klein, 2017). Studies have subsequently touted the benefits and ease of increasing meaning salience (Burrow \& Hill, 2013; King, Hicks, Krull, \& Del Gaiso, 2006; Masters, Ross, Hooker, \& Wooldridge, 2018). In all, research suggests that making meaning salient relates to meaning in life and has potential advantages for mental health (Hooker, Post, \& Sherman, 2020b). Together, this points to meaning salience as a promising way to alleviate suffering during times of isolation.

The current research aimed to increase meaning salience to facilitate well-being during the global COVID-19 pandemic. Specifically, we designed a longitudinal intervention designed to bring daily awareness to the meaningful moments in participants' lives. We did this during a week when most people were forced to stay home and had little access to normal 
avenues of finding meaning. We predicted that doing so would prevent an otherwise significant decrease in mental health.

\section{Meaning in Life}

Meaning in life is defined as a sense that one's life matters, makes sense, and has purpose (George \& Park, 2016; King, Heintzelman, \& Ward, 2016; Martela \& Steger, 2016; Schlegel \& Hicks, 2011). Some argue that finding meaning in life is at the core of human existence (Park, 2017). In general, researchers assert that a meaningful life is a life that one interprets as meaningful (Baumeister \& Landau, 2018). That is, experiencing meaning in life represents how individuals perceive their lives rather than how they actually live.

Having meaning in life, and thus engaging in meaningful activities, has its roots in the fulfillment of basic psychological needs (Deci \& Ryan, 2000) and leads to increased well-being (Hooker, Slattengren, Boyle, \& Sherman, 2019). In particular, meaning relates to numerous positive outcomes, including increased quality of life, life satisfaction, social support, and optimism (Guerra, Lencastre, Silva, \& Teixeira, 2017; Shiah, Chang, Chiang, Lin, \& Tam, 2013; Zika \& Chamberlain, 1992). Importantly, much of the research points to specific improvement in mental health (Halama \& Dědová, 2007; Moomal, 1999). Studies suggest that meaning negatively relates to depression (Vehling et al., 2011) and anxiety, and positively relates to outcomes such as vitality, social functioning, and positive emotions (Shiah et al., 2013). Meta-analyses also suggest a strong relationship between meaning and positive health outcomes (Pinquart, 2002).

\section{Meaning Salience}

Meaning salience is defined as an awareness of the meaning in one's life (Hooker, Post, \& Sherman, 2020b). Preliminary findings suggest that bringing awareness to one's meaning in life can directly impact perceptions of quality of life (Hooker, Masters, Vagnini, \& Rush, 2020a), and self-esteem (Taubman-Ben-Ari, 2011). Increased meaning salience also relates to increased motivation for participation in physical activity (Hooker \& Masters, 2018) and decreased sensations of burnout and fatigue in doctors (Hooker, Post, \& Sherman, 2020b). Furthermore, when meaning is salient it appears to decrease negative mental health outcomes such as depression and anxiety (Edwards \& Van Tongeren, 2020 ) while increasing positive affect and one's ability to cope with stressors (Miao \& Gan, 2019).

People may report that they have a general sense that their lives are meaningful, but at a given moment may not be aware of that meaning (Hooker, Masters, \& Park, 2018). As a result, they may not experience the subsequent positive outcomes of increased meaning in life. Researchers have recently begun investigating the benefits of increasing daily awareness of meaning. When meaning is made salient, the overall presence of meaning in life and well-being increases (Miao \& Gan, 2019; Newman, Nezlek, \& Thrash, 2017). Further, a review conceded that when people are asked to discover meaning in their everyday activities (Baumeister \& Landau, 2018), positive mental health outcomes increase.

\section{Present Study}

In the current study, we sought to develop a brief and accessible intervention aimed at increasing meaning salience to understand its effect on people's well-being in a time of social isolation. Given the strong relationship among various aspects of well-being and mental health (Capone \& Petrillo, 2018), we assert that increasing meaning salience will directly impact participants anxiety, depression, and stress. There is already some evidence suggesting that increasing meaning salience directly impacts mental health (Hooker et al., 2018) and one's ability to cope with stress (Miao \& Gan, 2019). For example, one study suggests that daily questionnaires result in a significant relationship between meaning and depression (Hadden \& Smith, 2019). However, these studies have yet to ask participants to apply meaning to daily events or examined meaning salience in a challenging context such as a global pandemic.

Life drastically changed for many due to the global COVID-19 (aka coronavirus) pandemic. This pandemic resulted in hundreds of thousands of deaths, mass unemployment, and strict restrictions on people and businesses worldwide. Mandatory social distancing measures intended to slow the virus resulted in increased feelings of depression and anxiety (Rajkumar, 2020). This is not surprising given that social distancing measures, in particular, are likely to directly affect many of the predictors of a meaningful life, including autonomy, competence, and beneficence (Martela \& Ryan, 2016). Similarly, research suggests that those who are experiencing current suffering frequently have substantially lower levels of meaning in life and mental health (Edwards \& Van Tongeren, 2020).

In this time of a global pandemic, it is especially important to care for the mental health of the world. As such, finding simple and easy ways to increase meaning in life is imperative for promoting mental health. To this end, we designed a longitudinal experiment to test the effects of meaning salience on mental health. In the current study, we attempt to make meaning salient by having people think about each aspect of their day and identifying the meaning in them. In all, we want people to attend to the meaning already present in their lives, thus making meaning salient to them. To accomplish this, we used daily diaries which research has found to be a reliable and effective way to increase daily awareness (Hülsheger, Alberts, Feinholdt, \& Lang, 2012; Kashdan \& McKnight, 2013; Scheck, Hoffmann, Proctor, \& Couillou, 2013). For one week, a group of participants reflected on their daily activities and the 
meaning gained from these activities while another group went about their week as normal. Mental health measures were employed before the week began and one week later, at the end of the experimental week.

\section{Method}

\section{Participants}

To obtain $95 \%$ power of detecting a medium effect size $(f=.15)$ at a .05 probability level, 107 participants were needed across our two groups. We rounded up to 120 total participants (60/group) to ensure sufficient statistical power. We used a database of prospective participants that had previously reported interest in participating in future studies conducted by our lab. All of them resided in the U.S.A. and spoke English fluently. Of the 401 invitations we sent, 153 unique participants responded to our invitation and completed at least a portion of the pre-test survey. Of these, a total of 135 unique participants provided complete data (i.e., completed both surveys).

To detect carelessness, we next examined several indicators available in the data (Nichols \& Edlund, 2020). We eliminated five participants who responded with identical responses (e.g., "4") at least 10 consecutive times (Johnson, 2005) and another 13 based on Qualtrics' fraud score. We next excluded 20 participants due to failing to correctly answer at least one of the 8 attention check questions embedded into the surveys (i.e., 4 each survey). Finally, we excluded one participant who reported currently living outside of the U.S.A. This left us with 96 U.S. resident participants that provided quality data for pre- and post-test administrations.

This final sample ranged in age from 22 to $69(M=34.45$, $s d=5.15)$. They largely identified as female $(92.7 \% ; 7.3 \%$ male), "White" (97.9\%; 2.1\% "Black/African American"), married (95.8\%), and had children (95.8\%). Almost all of them were currently employed (97.9\%) and had attended college (19.8\% some college; $3.1 \%$ two-year degree; $59.4 \%$ Bachelor's degree; $17.7 \%$ Master's degree). They mostly lived in urban $(60.4 \%)$ and suburban areas $(37.5 \% ; 2.1 \%$ rural) and had a variety of household incomes $(3.1 \% \$ 20-35 \mathrm{k}$; $28.1 \% \$ 35-50 \mathrm{k} ; 42.7 \% \$ 50-75 \mathrm{k} ; 22.9 \% \$ 75-100 \mathrm{k} ; 2.1 \%$ $\$ 100-150 \mathrm{k} ; 1.0 \%$ above $\$ 150 \mathrm{k})$.

\section{Procedure}

The study was approved by IntegReview IRB (protocol "CONNECT LONG). During the first weekend in April 2020 (i.e., the weekend of April 4, 2020), we emailed prospective participants. To avoid possible participant crosstalk (Edlund et al., 2014), we informed participants that the purpose of this study was "to understand people's daily activities." The link provided in the email directed participants to the informed consent in Qualtrics. Once they provided consent, they completed the pre-test survey. As soon as they completed the survey, the platform randomly assigned each participant to a condition and provided them with instructions for the week.

\section{Control $(n=51)$}

"In a week, we will send you another survey to complete..."

\section{Experimental $(n=45)$}

"Starting Monday, and continuing until Friday, we will send you text reminders three times per day to help you attend to your activities and the meaningfulness of them throughout the day. We will then send you a link each evening to complete your daily activity. In this activity, we will ask you to reflect on what you have done during the day and how meaningful each of those activities were...Next Saturday, we will send you another survey, similar to this one, to complete..."

The experimental group then completed a daily task for five days (Monday, April 6 -Friday, April 10) During the morning, afternoon, and evening on each day, participants additionally received the following text message on their phones:

"As you go through the next few hours of your day, please remember to attend to the activities that you are engaging in. In addition, make sure to think about how meaningful each of these moments are. We will ask about each of these daily moments when you reflect on your day later tonight."

Each night, they completed a modified version of the Meaningful Activity Participation Assessment (Eakman, Carlson, \& Clark, 2010).

A week after completing the pre-test (i.e., the weekend of April 11, 2020), we emailed all participants (in both conditions) the post-test survey. Among other measures, participants completed measures of mental health at pre- and posttest.

\section{Measures}

Daily Activities To provide a standardized way for participants to report their daily activities, we administered a slightly modified version of the Meaningful Activity Participation Assessment (Eakman et al., 2010). At the end of each day, they saw a list of 44 activities (e.g., emailing, using public transportation, cooking) plus two fill-in-the-blank options that allowed them to 
list activities that were not included in the list. After checking the box next to each of the activities that they engaged in that day, participants reported the number of minutes they engaged in each activity. Finally, those in the experimental group rated how meaningful each reported activity was to them that day using a five-point Likert scale ( $1=$ "Not at all meaningful", $5=$ "Extremely meaningful").

Mental Health We used the Depression Anxiety Stress Scales to measure various aspects of mental health (DASS; Lovibond \& Lovibond, 1995). Participants responded to 21 items on a four-point scale $(0=$ Never; $3=$ Almost always $)$. An example item is "I found it hard to wind down" (Anxiety: $\alpha_{\text {pre }}=.88$, $\alpha_{\text {post }}=.91$, Depression: $\alpha_{\text {pre }}=.90, \alpha_{\text {post }}=.91$, Stress: $\alpha_{\text {pre }}=.88, \alpha_{\text {post }}=.91$ ).

COVID-19 We additionally asked participants about their current situation related to COVID-19. This included what local restrictions were implemented, how they have been impacted by them, and what measures they were personally taking due to COVID-19.

Demographics To better understand our sample, we also asked questions related to age, gender, race, relationship status, education, living situation, employment status, and income.

\section{Analysis}

We used the R statistical program, with associated packages, to analyze the data (R Core Team, 2017). We first performed t-tests to examine baseline mental health differences between conditions. To examine the direct effects of meaning salience on mental health, over time, we then performed three multilevel models with both time points (pre, post), condition (control, experimental), and the interaction of the two as predictor variables with each of the mental health outcomes as dependent variables. In these models, time was the level 1 variable and was nested within participants (the level 2 variable). In all, we analyzed 192 responses across 96 participants.

\section{Results}

Before we began our primary analyses, we examined participants' responses regarding how their lives were affected by COVID-19 and the corresponding restrictions. At the beginning of the week, $54.2 \%$ of participants reported engaging in a total self-quarantine, $36.9 \%$ staying home as much as possible, and only $6.3 \%$ that they were not going into work, but that everything else remained the same. At the end of the week, that changed to $51.0 \%$ engaging in a total self-quarantine and $49.0 \%$ staying home as much as possible. Regarding the extent to which the governmental restrictions had affected their lives during the experimental week, $62.5 \%$ reported that they were impacted "a great deal" over the past week, $34.4 \%$ were affected "a lot", and $3.1 \%$ were affected "a moderate amount". None selected the "a little" or "none at all" options. Quantifying this question, ( $1=$ "none at all", $5=$ "a great deal") resulted in an alarmingly high mean of $4.59(s d=0.55)$.

We also examined the activities that participants in the experimental condition reported engaging in. Sleep, working alone, watching TV, working with others, and cooking were the top five activities participants engaged in. The activities with the highest meaning ratings were working alone, meeting with someone new, cooking, sleeping, and playing games alone. The lowest ratings were reported for traveling, physical exercise with others, writing letters/cards, socializing, and instant messaging/ texting.

We next compared the baseline mental health of participants in each condition. The results suggested that participants in the control group began the intervention period with similar levels of anxiety, $t(88.68)=0.87, p=.39$ (Control: $M=2.17$, $s d=0.57$, Exp: $M=2.06, s d=0.64)$, depression, $t(88.82)=$ 1.00, $p=.32$ (Control: $M=2.23, s d=0.58$, Exp: $M=2.10, s d-$ $=0.66)$, and stress, $t(90.22)=1.69, p=.09$ (Control: $M=$ $2.27, s d=0.54$, Exp: $M=2.08, s d=0.58$ ). This suggests that our random assignment of participants to conditions successfully distributed people based on their mental health.

To test of hypotheses, we next performed multi-level modeling for each of the three mental health outcomes. The first model examined anxiety as the outcome. As hypothesized, this resulted in a significant interaction between time and condition, $b=-.23$, se $=.06, t(94)=-3.83, p<.001$ (see Fig. 1). To dissect this interaction, we computed estimated marginal means to compare both groups across time. In the control condition, participants reported higher anxiety during the post-test, $M=2.30, \mathrm{se}=.09$, than during the pre-test, $M=$ 2.17, $s e=.09, t=-3.30, p=.001, d=.44$. In contrast, the experimental condition reported less anxiety after the experimental week, $M=1.96$, se $=.09$, than before the week began, $M=2.06, s e=.09, t=2.15, p=.03, d=.33$. This suggests that people were generally becoming more anxious during this troubling week. However, making meaning salient was able to reverse this effect and reduce the amount of anxiety participants experienced over the week.

We next performed the same analyses for depression as the outcome. This again resulted in a significant interaction between time and condition, $b=-.15$, se $=.06, t(94)=-2.53, p=.01$ (see Fig. 2). The trends were similar to those found for anxiety. Participants in the control group reported more depression during the post-test, $M=2.34$, se $=.09$, than during the pre-test, $M=2.23, s e=.09, t=-2.89, p=.005, d=.40$. In contrast, for the experimental group, depression scores decreased from pretest, $M=2.10, s e=.09$, to post-test, $M=2.07$, $s e=.09$, but the difference was nonsignificant, $t=0.75, p=.45, d=.10$. 


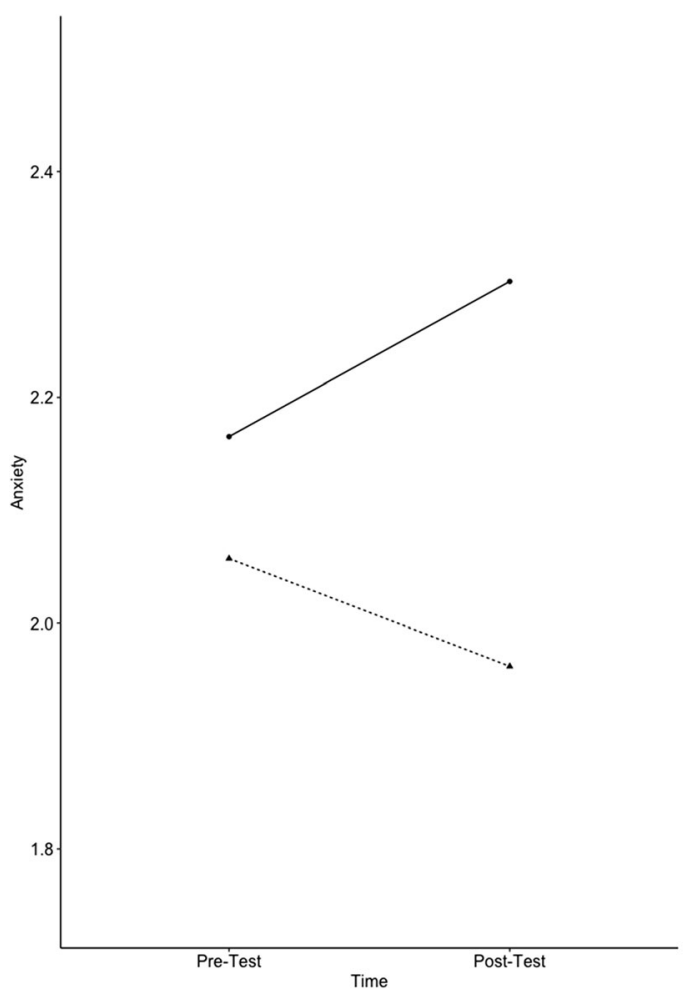

Fig. 1 The Effect of Condition on Anxiety across Time

A pattern similar to depression emerged for stress. A significant interaction between time and condition again resulted, $b=$ -.23 , se $=.07, t(94)=-3.44, p<.001$ (see Fig. 3). Specifically, the control condition became significantly more stressed during the week (Pre: $M=2.27$, se $=.08$; Post: $M=2.44$, $s e=.08$ ), $t=$ $-3.70, p=.005, d=.65$, while the experimental condition decreased slightly but not significantly (Pre: $M=2.08$, se $=.09$; Post: $M=2.02$, se =.09), $t=1.25, p=.22, d=.16$. In general, the control group became more anxious, depressed, and stressed during this tough quarantine week. However, making meaning salient appeared to guard against this effect.

The full dataset and analysis code are available upon request from the first author.

\section{Discussion}

Research on meaning salience has just now begun to explore the benefits of a momentary awareness of one's meaning (Hooker \& Masters, 2018; Hooker, Masters, et al., 2020a). Despite theorizing about both the physical and psychological benefits of meaning salience, research has yet to examine how a daily awareness of meaning can affect mental health. Given the challenging context of the COVID-19 pandemic and subsequent social isolation, we believed it was more important than ever to understand if meaning salience can additionally benefit mental health. To this end, we conducted a

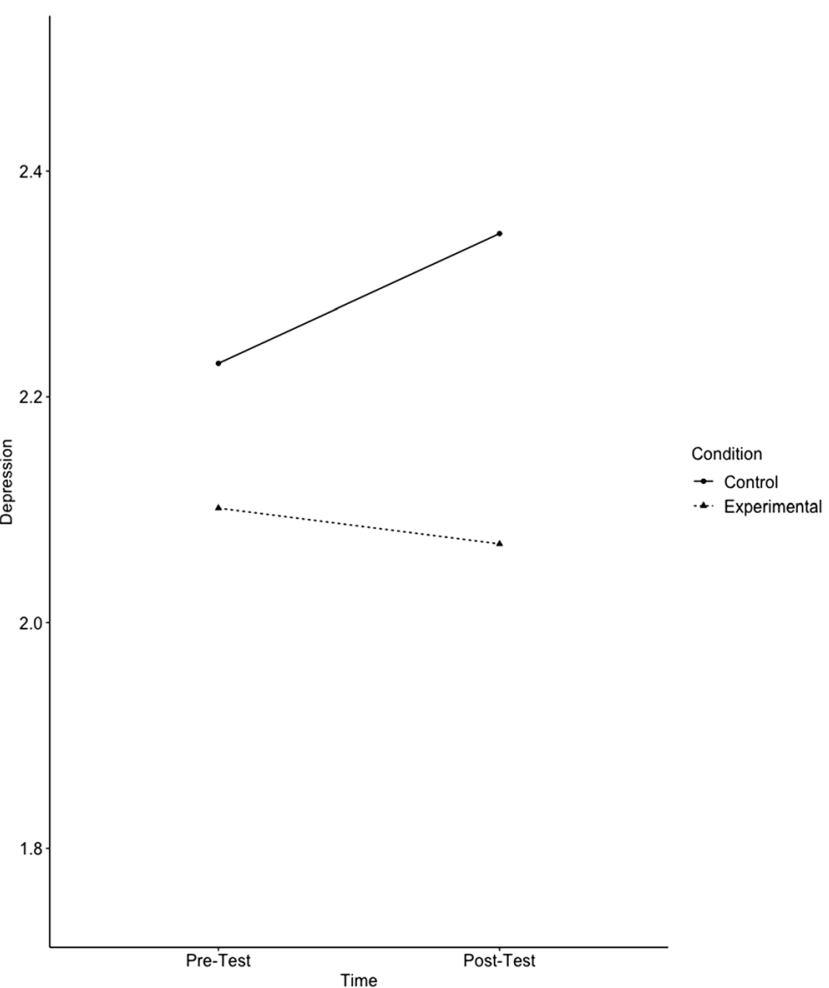

Fig. 2 The Effect of Condition on Depression across Time

longitudinal experiment over the course of one week. The intervention group reported on their daily activities and meaning associated with them while the control group lived life as normal.

Alarmingly, the control group grew significantly more anxious, depressed, and stressed during this week. To put this into context, our experimental period was the week of April 5, 2020. Although the virus had been spreading for months around the world, quarantine restrictions had just begun in many U.S. states. Many of these extended well into May and even June, and many people in the U.S.A. were socially isolated during this entire period. Studies throughout history have shown drastic changes in mental health during times of crisis (Uutela, 2010). If the data from our study is any indication of the overall decrease in the population's mental health throughout this period, it suggests strong effects of these "stay at home" orders on mental health.

Participants in the experimental group did not experience these same decreases in mental health. In contrast, they became significantly less anxious and trended toward less depression and stress at the end of the week. Given the strong trends toward negative mental health in the control group, these findings are both promising and provide hope that simple options exist to deal with mental health problems both in "normal" times and times that are especially challenging. By spending only a few minutes every day focusing on the activities they engaged in each day, the time spent on each, and the 


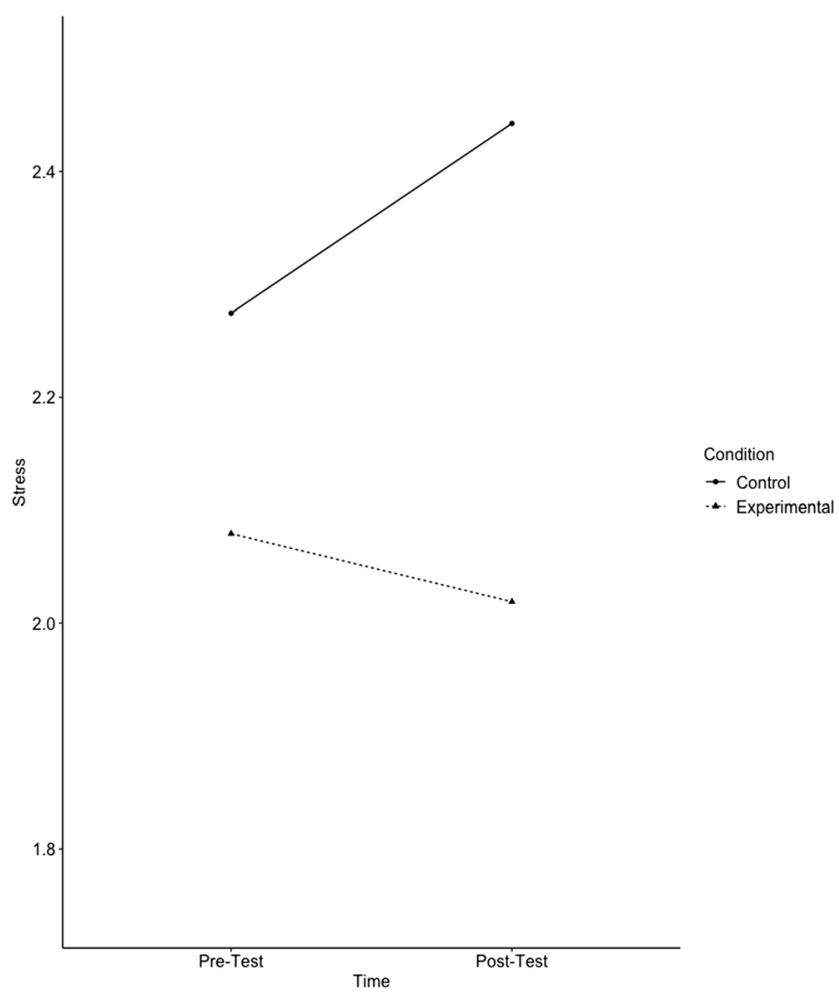

Fig. 3 The Effect of Condition on Stress across Time

meaning gained from each that day, people were able to increase their mental health during a week when most people's mental health was decreasing.

Our findings are consistent with past research in both daily meaning and gratitude research. One study found daily reflection on things that you are grateful for can increase mental health, even among those who have experienced trauma (Kashdan, Uswatte, \& Julian, 2006). Other research indicates the usefulness of making meaning salient for preventing burnout and fatigue in high stress environments (Hooker, Post, \& Sherman, 2020b). Together with our current results, evidence is mounting that meaning salience is a potentially useful tool for maintaining and even promoting mental health outcomes in times of crisis.

\section{Limitations and Future Directions}

As with any research, this study was not without its limitations. First, despite our efforts to obtain a larger sample, our efforts resulted in just under 100 participants. As such, future research should examine these effects in larger samples. Similarly, our sample was relatively homogeneous (i.e., predominantly white, married females with children), and future research would benefit from efforts aimed at collecting data from a more representative sample of the population. Despite the importance of examining these effects during COVID-19 restrictions, it would also be beneficial to understand what they look like when people are able to live life as they normally do. During "normal" times, mental health should not decline over the course of a week. The question then becomes if the intervention still benefits mental health significantly more compared to a control group.

Future research should additionally investigate the impacts of our meaning salience intervention in the context of other forms of crisis. This could be particularly useful in the context of other challenging situations worldwide. For example, in the context of unstable governments or refugee crises, finding the meaning in everyday moments could be very useful. In fact, meta-analytic results point to the need to attend to mental health in these contexts (Porter \& Haslam, 2005). A meaning salience intervention could help people identify the meaningful experiences in an otherwise challenging and chaotic life and prevent what might otherwise be decreased overall mental health. The benefits of an intervention aimed at increasing meaning salience, such as the one used in the current study, can likely be realized in many contexts due to its easy implementation across multiple contexts.

Finally, more research is needed on meaning salience and on developing effective interventions that increase it. To begin, research would benefit from further consideration of how to operationalize meaning salience in research contexts. One especially important avenue would be to develop and psychometrically examine a measure of meaning salience. Recently, the Thoughts of Meaning Scale (TOMS) was developed for use in a study related to meaning salience (Hooker \& Masters, 2018), but it has yet to be psychometrically evaluated in a way that provides researchers with confidence regarding is reliability and validity. This would be a good place for research to begin. This will then help future intervention research evaluate the effectiveness of interventions by using the TOMS or another measure of meaning salience as a metric for determining intervention success.

\section{Conclusion}

The current study examined the effects of a simple weeklong intervention on mental health during the COVID-19 pandemic. Those reporting how much meaning they derived from their daily activities reported less anxiety and marginally less depression and stress a week later. In contrast, the control group reported significantly worse anxiety, depression and stress from pre- to post-test. Considering the challenging context of this experimental week, results suggest that this simple daily activity is a promising option for those going through tough times and may prevent otherwise deteriorating mental health from prolonged isolation.

Data Availability (Data Transparency) The data is available upon request from the first author. 
Code Availability (Software Application or Custom Code) The code is available upon request from the first author.

\section{Declarations}

Conflicts of Interest The authors declare no conflicts of interest

\section{References}

Baumeister, R. F., \& Landau, M. J. (2018). Finding the meaning of meaning: Emerging insights on four grand questions. Review of General Psychology, 22(1), 1-10. https://doi.org/10.1037/gpr0000145.

Burrow, A. L., \& Hill, P. L. (2013). Derailed by diversity? Purpose buffers the relationship between ethnic composition on trains and passenger negative mood. Personality and Social Psychology Bulletin, 39(12), 1610-1619. https://doi.org/10.1177/0146167213499377.

Capone, V., \& Petrillo, G. (2018). Mental health in teachers: Relationships with job satisfaction, efficacy beliefs, burnout and depression. Current Psychology, 39, 1-10. https://doi.org/10.1007/ s12144-018-9878-7.

Deci, E. L., \& Ryan, R. M. (2000). The " what " and " why " of goal pursuits: Human needs and the self-determination of behavior. Psychological Inquiry, 11(4), 227-268. https://doi.org/10.1207/ S15327965PLI1104.

Eakman, A. M., Carlson, M. E., \& Clark, F. A. (2010). The meaningful activity participation assessment: A measure of engagement in personally valued activities. The International Journal of Aging and Human Development, 70(4), 299-317. https://doi.org/10.2190/AG. 70.4.b.

Edlund, J. E., Nichols, A. L., Okdie, B. M., Guadagno, R. E., Eno, C. A., Heider, J. D., Hansen, E. J., Sagarin, B. J., Blackhart, G., Cottrell, C. A., \& Wilcox, K. T. (2014). The prevalence and prevention of crosstalk: A multi-institutional study. The Journal of Social Psychology, 154(3), 181-185. https://doi.org/10.1080/00224545. 2013.872596.

Edwards, M. E., \& Van Tongeren, D. R. (2020). Meaning mediates the association between suffering and well-being. Journal of Positive Psychology, 15, 1-12. https://doi.org/10.1080/17439760.2019. 1651890.

George, L. S., \& Park, C. L. (2016). Meaning in life as comprehension, purpose, and mattering: Toward integration and new research questions. Review of General Psychology, 20(3), 205-220. https://doi. org/10.1037/gpr0000077.

Guerra, M. P., Lencastre, L., Silva, E., \& Teixeira, P. M. (2017). Meaning in life in medical settings: A new measure correlating with psychological variables in disease. Cogent Psychology, 59(1), 1-12. https:// doi.org/10.1080/23311908.2017.1286747.

Hadden, B. W., \& Smith, C. V. (2019). I gotta say, today was a good (and meaningful) day: Daily meaning in life as a potential basic psychological need. Journal of Happiness Studies, 20(1), 185-202. https:// doi.org/10.1007/s10902-017-9946-y.

Halama, P., \& Dědová, M. (2007). Meaning in life and hope as predictors of positive mental health: Do they explain residual variance not predicted by personality traits? Studia Psychologica, 49(3), 191200

Heintzelman, S. J. (2018). Eudaimonia in the contemporary science of subjective well-being: Psychological well-being, self- determination, and meaning in life. In E. Diener, S. Oishi, \& L. Tay (Eds.), Handbook of well-being. DEF Publishers. nobascholar.com.

Heintzelman, S. J., Kushlev, K., Lutes, L. D., Wirtz, D., Kanippayoor, J. M., Leitner, D., Oishi, S., \& Diener, E. (2020). ENHANCE:
Evidence for the efficacy of a comprehensive intervention program to promote subjective well-being. Journal of Experimental Psychology: Applied, 26(2), 360-383. https://doi.org/10.1037/ xap0000254.

Hooker, S. A., \& Masters, K. S. (2018). Daily meaning salience and physical activity in previously inactive exercise initiates. Health Psychology, 37(4), 344-354. https://doi.org/10.1037/hea0000599.

Hooker, S. A., Masters, K. S., \& Park, C. L. (2018). A meaningful life is a healthy life: A conceptual model linking meaning and meaning salience to health. Review of General Psychology, 22(1), 11-24. https://doi.org/10.1037/gpr0000115.

Hooker, S. A., Masters, K. S., Vagnini, K. M., \& Rush, C. L. (2020a). Engaging in personally meaningful activities is associated with meaning salience and psychological well-being. Journal of Positive Psychology, 1-11. https://doi.org/10.1080/17439760. 2019.1651895.

Hooker, S. A., Post, R. E., \& Sherman, M. D. (2020b). Awareness of meaning in life is protective against burnout among family physicians. Family Medicine, 52(1), 11-16. https://doi.org/10.22454/ FamMed.2019.562297.

Hooker, S. A., Slattengren, A. H., Boyle, L., \& Sherman, M. D. (2019). Values-based behavioral activation for chronic pain in primary care: A pilot study. Journal of Clinical Psychology in Medical Settings, 0123456789. https://doi.org/10.1007/s10880-019-09655-x.

Hülsheger, U. R., Alberts, H. J. E. M., Feinholdt, A., \& Lang, J. W. B. (2012). Benefits of mindfulness at work: The role of mindfulness in emotion regulation, emotional exhaustion, and job satisfaction. Journal of Applied Psychology, 98(2), 310-325. https://doi.org/10. 1037/a0031313.

Johnson, J. A. (2005). Ascertaining the validity of individual protocols from web-based personality inventories. Journal of Research in Personality, 39(1), 103-129. https://doi.org/10.1016/j.jrp.2004.09. 009.

Kashdan, T. B., \& McKnight, P. E. (2013). Commitment to a purpose in life: An antidote to the suffering by individuals with social anxiety disorder. Emotion, 13(6), 1150-1159. https://doi.org/10.1037/ a0033278.

Kashdan, T. B., Uswatte, G., \& Julian, T. (2006). Gratitude and hedonic and eudaimonic well-being in Vietnam war veterans. Behaviour Research and Therapy, 44(2), 177-199. https://doi.org/10.1016/j. brat.2005.01.005.

King, L. A., Heintzelman, S. J., \& Ward, S. J. (2016). Beyond the search for meaning: A contemporary science of the experience of meaning in life. Current Directions in Psychological Science, 25(4), 211216. https://doi.org/10.1177/0963721416656354.

King, L. A., Hicks, J. A., Krull, J. L., \& Del Gaiso, A. K. (2006). Positive affect and the experience of meaning in life. Journal of Personality and Social Psychology, 90(1), 179-196. https://doi.org/10.1037/ 0022-3514.90.1.179.

Klein, N. (2017). Prosocial behavior increases perceptions of meaning in life. Journal of Positive Psychology, 12(4), 354-361. https://doi.org/ 10.1080/17439760.2016.1209541.

Lovibond, S., \& Lovibond, P. (1995). Manual for the depression anxiety \& stress scales (2nd ed.). Sydney: Psychology Foundation.

Martela, F., \& Ryan, R. M. (2016). The benefits of benevolence: Basic psychological needs, beneficence, and the enhancement of well-being. Journal of Personality, 84(6), 750-764. https://doi.org/10. 1111/jopy.12215.

Martela, F., \& Steger, M. F. (2016). The three meanings of meaning in life: Distinguishing coherence, purpose, and significance. The Journal of Positive Psychology, 11(5), 531-545. https://doi.org/10. 1080/17439760.2015.1137623.

Masters, K. S., Ross, K. M., Hooker, S. A., \& Wooldridge, J. L. (2018). A psychometric approach to theory-based behavior change intervention development: Example from the Colorado meaning- activity 
project. Annals of Behavioral Medicine, 52(6), 463-473. https://doi. org/10.1093/abm/kay023.

Miao, M., \& Gan, Y. (2019). How does meaning in life predict proactive coping? The self-regulatory mechanism on emotion and cognition. Journal of Personality, 87(3), 579-592. https://doi.org/10.1111/ jopy.12416.

Moomal, Z. (1999). The relationship between meaning in life and mental well-being. South Africa Journal of Psychology, 29(1), 36-41.

Newman, D. B., Nezlek, J. B., \& Thrash, T. M. (2017). The dynamics of searching for meaning and presence of meaning in daily life. Journal of Personality, 0(0), 1-11. https://doi.org/10.1111/jopy. 12321.

Nichols, A. L., \& Edlund, J. E. (2020). Why don't we care more about carelessness? Understanding the causes and consequences of careless participants. International Journal of Social Research Methodology, 23(6), 625-638. https://doi.org/10.1080/13645579. 2020.1719618.

Park, C. L. (2017). Unresolved tensions in the study of meaning in life. Journal of Constructivist Psychology, 30(1), 69-73. https://doi.org/ 10.1080/10720537.2015.1119083.

Pinquart, M. (2002). Creating and maintaining purpose in life in old age: A meta-analysis. Ageing International, 27(2), 90-114. https://doi. org/10.1007/s12126-002-1004-2.

Porter, M., \& Haslam, N. (2005). Predisplacement and postdisplacement factors associated with mental health of refugees and internally displaced persons: A meta-analysis. JAMA, 294(5), 602-612. https://doi.org/10.1001/jama.294.5.602.

R Core Team (2017). R: A language and environment for statistical computing. R Foundation for Statistical Computing, Vienna, Austria. URL https://www.R-project.org/.

Rajkumar, R. P. (2020). COVID-19 and mental health: A review of the existing literature. Asian Journal of Psychiatry, 52, 102066. https:// doi.org/10.1016/j.ajp.2020.102066.
Scheck, A. M., Hoffmann, N. G., Proctor, S. L., \& Couillou, R. J. (2013). Interactive journaling as a brief intervention for level-II DUI and DWI offenders. Journal of Alcohol and Drug Education, 57(3), 66-85.

Schlegel, R. J., \& Hicks, J. A. (2011). The true self and psychological health: Emerging evidence and future directions. Social and Personality Psychology Compass, 5(12), 989-1003. https://doi. org/10.1111/j.1751-9004.2011.00401.x.

Shiah, Y. J., Chang, F., Chiang, S. K., Lin, I. M., \& Tam, W. C. C. (2013). Religion and health: Anxiety, religiosity, meaning of life and mental health. Journal of Religion and Health, 54(1), 35-45. https://doi. org/10.1007/s10943-013-9781-3.

Taubman-Ben-Ari, O. (2011). Is the meaning of life also the meaning of death? A terror management perspective reply. Journal of Happiness Studies, 12(3), 385-399. https://doi.org/10.1007/ s10902-010-9201-2.

Uutela, A. (2010). Economic crisis and mental health. Current Opinion in Psychiatry, 23(2), 127-130. https://doi.org/10.1097/YCO. 0b013e328336657d.

Vehling, S., Lehmann, C., Oechsle, K., Bokemeyer, C., Krüll, A., Koch, U., \& Mehnert, A. (2011). Global meaning and meaning-related life attitudes: Exploring their role in predicting depression, anxiety, and demoralization in cancer patients. Supportive Care in Cancer, 19(4), 513-520. https://doi.org/10.1007/s00520-010-0845-6.

Zika, S., \& Chamberlain, K. (1992). On the relation between meaning in life and psychological well-being. British Journal of Psychology, 83, 133-145. http://onlinelibrary.wiley.com/doi/10.1111/j.20448295.1992.tb02429.x/full.

Publisher's Note Springer Nature remains neutral with regard to jurisdictional claims in published maps and institutional affiliations. 